\title{
Twist-3 asymmetries in proton-proton collisions
}

\author{
Yuji Koike* \\ Department of Physics, Niigata University, Ikarashi 2-no-cho, Nishi-ku, Niigata 950-2181, \\ Japan \\ E-mail: koike@phys.sc.niigata-u.ac.jp
}

\section{Daniel Pitonyak}

Division of Science, Penn State University-Berks, Readiing, PA 19610, USA

E-mail: dpitonyak@quark.phy.bnl.gov

\section{Kenta Yabe}

Graduate School of Science and Technology, Niigata University, Ikarashi 2-no-cho, Nishi-ku, Niigata 950-2181, Japan

E-mail: k.yabe.hadron@gmail.com

\section{Shinsuke Yoshida}

Key Laboratory of Quark and Lepton Physics (MOE) and Institute of Particle Physics, Central China Normal University, Wuhan 430079, China

E-mail: shinyoshida85@gmail.com

\begin{abstract}
We discuss two twist-3 spin asymmetries in $p p$ collisions. One is the transverse polarization of hyperons produced in unpolarized $p p$ collisions, and the other is the spin asymmetry for the hadron production in the collision between the longitudinally and transversely polarized protons. The former is a naively " $T$-odd" observable, while the latter is a naively " $T$-even" one, and this leads to inherently different form of the corresponding twist- 3 cross sections. Since both asymmetries occur as a result of multi-parton correlations either in the initial protons or in the fragmentation process, they play a complementary role to test the theoretical framework and to give an insight on the hadron structure. Measurement of these asymmetries at RHIC will be particularly interesting.
\end{abstract}

QCD Evolution 2016

May 30-June 03, 2016

National Institute for Subatomic Physics (Nikhef), Amsterdam

\footnotetext{
* Speaker.

${ }^{\dagger}$ Present address: Theoretical Division, Los Alamos National Laboratory, Los Alamos, NM 87545, USA.
} 


\section{Introduction}

In this paper, we study two twist-3 spin asymmetries in proton-proton collisions. The first one is the large hyperon polarization $(\sim$ a few ten $\%)$ in unpolarized $p p$ collisions which has been known since 1970s [1] $]^{1}$,

$$
p(P)+p\left(P^{\prime}\right) \rightarrow H^{\uparrow}\left(P_{h}, S_{\perp}\right)+X
$$

where the momentum and the spin vector for each baryon is shown. Perturbative QCD at twist-2 level can not give rise to such large polarization [2], and therefore clarification of its origin has been a big challenge for QCD theorists. In the collinear factorization the polarization can occur as a twist-3 effect and the cross section receives two types of contributions: (i) Twist- 3 distribution function (quark-gluon correlation function) in one of the unpolarized protons combined with the twist-2 transversity fragmentation function for $H^{\uparrow}$ and the twist-2 parton density in another proton, and (ii) Twist-3 fragmentation function for the polarized $H$ combined with the unpolarized parton densities for the initial protons. In our recent paper [3], we completed the twist- 3 cross section for (i) in the leading-order (LO) with respect to the QCD coupling constant. Reflecting the naively " $T$-odd" nature of the polarization, the twist-3 cross section for (i) occurs as pole contributions in the hard part. It turned out that the soft-fermion-pole (SFP) contribution vanishes and only the derivative term of the soft-gluon-pole (SGP) contribution survives for (i). In Sec. 2, we discuss these features following [3].

The second one is the longitudinal and transverse spin asymmetry $A_{L T}$ in the hadron (typically pion) production in $p p$ collisions:

$$
p^{\uparrow}\left(P, S_{\perp}\right)+\vec{p}\left(P^{\prime}, \Lambda\right) \rightarrow h\left(P_{h}\right)+X,
$$

where $S_{\perp}$ is the spin vector of the transversely polarized nucleon $A$, and $\Lambda$ is the helicity of the longitudinally polarized nucleon $B$. The twist- 3 cross section for (1.2) consists of three parts:

$$
\begin{aligned}
d \sigma\left(P_{h}, S_{\perp}, \Lambda\right) & =H \otimes f_{a / A(3)} \otimes f_{b / B(2)} \otimes D_{h / c(2)} \\
& +H^{\prime} \otimes f_{a / A(2)} \otimes f_{b / B(3)} \otimes D_{h / c(2)} \\
& +H^{\prime \prime} \otimes f_{a / A(2)} \otimes f_{b / B(2)} \otimes D_{h / c(3)},
\end{aligned}
$$

where $f_{a / A(3)}$ represents twist-3 distribution function of parton species $a$ in the nucleon $A$, and $D_{h / c(2)}$ is the twist-2 fragmentation function for $c \rightarrow h$, and likewise for other functions. The LO cross section for the first term was derived in [4]. In our recent papers, we derived the LO cross section for the second [5] and the third [6] terms, which completed the cross section for (1.2). Unlike transverse single spin asymmetries, $A_{L T}$ is naively " $T$-even", which leads to inherently different form of the twist- 3 cross section. Therefore study on $A_{L T}$ can shed light on different aspect of hadron structure and can provide further test of the formalism. There are some reasons that the second and the third terms in (1.3) are as important as the first term: For $A_{L T}$ in the DrellYan process, there are two twist-3 terms corresponding to the first and the second terms in (1.3) with $D_{h / c(2)}$ omitted. An analysis of $A_{L T}$ of Drell-Yan shows the second term is as important as

\footnotetext{
${ }^{1}$ We collectively use the notation $H$ for hyperons.
} 
the first term when one integrates over the transverse momentum of the lepton pair [7]. For $A_{N}$ of $p^{\uparrow} p \rightarrow \pi X$, it's been shown that the twist- 3 fragmentation contribution may be the main cause of the asymmetry [8], from which we expect the third term in (1.3) may be as important as the others. Accordingly, study of all three terms in (1.3) is needed. In Sec. 3, we will present the cross sections for the second and third terms following $[5,6]$.

\section{Hyperon polarization from unpolarized $p p$ collision}

Here we are interested in the contribution to (1.1) from the twist-3 distribution in the unpolarized nucleon $E_{F}\left(x_{1}, x_{2}\right)$ and the twist-2 transversity fragmentation function $H_{1}(z)$ for $H^{\uparrow}$. The former is defined as $[9,10]$

$$
\int \frac{d \lambda}{2 \pi} \int \frac{d \mu}{2 \pi} e^{i \lambda x_{1}} e^{i \mu\left(x_{2}-x_{1}\right)}\left\langle P\left|\bar{\psi}_{j}(0) g F^{\alpha n}(\mu n) \psi_{i}(\lambda n)\right| P\right\rangle=\frac{M_{N}}{4} \varepsilon^{\alpha \beta n p}\left(\gamma_{5} \gamma_{\beta} p\right)_{i j} E_{F}\left(x_{1}, x_{2}\right)+\cdots,
$$

where $\psi_{i}$ is a quark field with spinor index $i$, and we introduced two lightlike vectors $p$ and $n$ which satisfy $P^{\mu}=p^{\mu}+\left(M_{N}^{2} / 2\right) n^{\mu}$ and $p \cdot n=1$ with the only nonzero components $p^{+}=P^{+}$ and $n^{-}$for the nucleon moving in the $+z$-direction. The nucleon mass $M_{N}$ is introduced to define the function $E_{F}\left(x_{1}, x_{2}\right)$ as dimensionless, and $\varepsilon^{\alpha \beta n p} \equiv \varepsilon^{\alpha \beta \mu v} n_{\mu} p_{v}$. From hermiticity and $P T$ invariance, $E_{F}\left(x_{1}, x_{2}\right)$ is real and symmetric: $E_{F}\left(x_{1}, x_{2}\right)=E_{F}\left(x_{2}, x_{1}\right) . H_{1}(z)$ is defined as [10]

$$
\frac{1}{N} \sum_{X} \int \frac{d \lambda}{2 \pi} e^{i \frac{\lambda}{z}}\left\langle 0\left|\psi_{i}(\lambda w)\right| H\left(P_{h}, S_{\perp}\right) X\right\rangle\left\langle H\left(P_{h}, S_{\perp}\right) X\left|\bar{\psi}_{j}(0)\right| 0\right\rangle=\left(\gamma_{5} \S_{\perp} p_{c}\right)_{i j} H_{1}(z)+\cdots,
$$

where $N=3$ is the number of colors for a quark, the hyperon momentum $P_{h}$ is decomposed as $P_{h}^{\mu}=p_{h}^{\mu}+\left(M_{h}^{2} / 2\right) w^{\mu}\left(M_{h}\right.$ is the hyperon mass) with two lightlike vectors $p_{h}$ and $w$ satisfying $p_{h} \cdot w=1$, and $p_{c} \equiv p_{h} / z$ is the momentum of the quark fragmenting into $H^{\uparrow}$. The combination of the two chiral-odd functions $E_{F}\left(x_{1}, x_{2}\right)$ and $H_{1}(z)$ can generate the polarization of the hyperon.

The twist- 3 cross section from $E_{F}\left(x_{1}, x_{2}\right)$ and $H_{1}(z)$ occurs as a pole contribution in the partonic hard part. They are classified as the soft-gluon-pole (SGP) and the soft-fermion-pole (SFP) which fix the momentum fraction of $E_{F}\left(x_{1}, x_{2}\right)$ at $x_{1}=x_{2}$ and $x_{i}=0(i=1$ or 2$)$, respectively. The SGP contribution was calculated in $[10,11]$, while the SFP contribution was not studied yet. In general, SGP gives rise to the contribution from $E_{F}(x, x)$ and its derivative $d E_{F}(x, x) / d x$. For this process, however, it was found that the hard factor for the nonderivative term vanishes [11] and only the derivative term survives in the cross section. This is in contrast to the case of the twist-3 distribution contribution to the single spin asymmetry for $p^{\uparrow} p \rightarrow h X$, where the derivative and the nonderivative terms of the SGP function $G_{F}(x, x)$ appears with a common hard factor, i.e., they appear in the combination of $x \frac{d}{d x} G_{F}(x, x)-G_{F}(x, x)$ [12]. The origin of this simple form was clarified based on the "master formula" which connects the SGP partonic hard cross section to a $2 \rightarrow 2$ scattering cross section [13]. Here we develop a "master formula" for $p p \rightarrow H^{\uparrow} X$ and clarify the origin of different type of simplification for the SGP cross section. We also compute the SFP contribution and complete the LO cross section for the twist-3 cross section.

We first discuss the SGP contribution. Applying the formalism developed in [14, 15], one can obtain the SGP contribution to $p p \rightarrow H^{\uparrow} X$ from the following formula:

$$
P_{h}^{0} \frac{d \Delta \sigma^{\mathrm{SGP}}}{d^{3} P_{h}}=\frac{i M_{N}}{64 \pi^{2} s} \int \frac{d x^{\prime}}{x^{\prime}} f_{1}\left(x^{\prime}\right) \int \frac{d z}{z^{2}} H_{1}(z)
$$




$$
\times\left.\int \frac{d x_{1}}{x_{1}} \int d x_{2} E_{F}\left(x_{1}, x_{2}\right) \varepsilon^{\alpha \beta n p} \frac{\partial}{\partial k_{2}^{\alpha}}\left(S_{\lambda \beta}^{\mathrm{I}}\left(k_{1}, k_{2}\right) p^{\lambda}+S_{\lambda \beta}^{\mathrm{F}}\left(k_{1}, k_{2}\right) p^{\lambda}\right)\right|_{k_{i}=x_{i} p}
$$

where $s=\left(p+p^{\prime}\right)^{2}$ is the center of mass energy squared, $f_{1}\left(x^{\prime}\right)$ is the unpolarized parton density, and the partonic hard parts $S_{\lambda \beta}^{\mathrm{I}, \mathrm{F}}\left(k_{1}, k_{2}\right) p^{\lambda}$ are obtained from diagrams shown in Fig. 1 by taking the spinor- and color- traces with the appropriate projections for the distribution and fragmentation functions. $S_{\lambda \beta}^{\mathrm{I}}$ and $S_{\lambda \beta}^{\mathrm{F}}$ correspond to the initial-state-interaction (ISI) and the finalstate-interaction (FSI), respectively. In $S_{\lambda \beta}^{\mathrm{I}, \mathrm{F}}\left(k_{1}, k_{2}\right)$, the Lorentz index $\lambda$ corresponds to that for the coherent gluon line with the momentum $k_{2}-k_{1}$ in Fig. 1 , and $\beta$ is for the projection of $E_{F}\left(x_{1}, x_{2}\right)$ in (2.1).

Following the procedure described in $[13,16,17]$, one obtains the hard part as

$$
\begin{aligned}
\left.\varepsilon^{\alpha \beta n p} \frac{\partial S_{\lambda \beta}^{\mathrm{I}}\left(k_{1}, k_{2}\right) p^{\lambda}}{\partial k_{2}^{\alpha}}\right|_{k_{i}=x_{i} p} ^{\mathrm{SGP}}= & {\left[\frac{-1}{x_{2}-x_{1}+i \varepsilon}\right]^{\text {pole }} \varepsilon^{\alpha \beta n p} S_{\perp}^{\gamma} \frac{d}{d\left(x^{\prime} p^{\prime \alpha}\right)} \widehat{S}_{\beta \gamma}^{\mathrm{I}}\left(x_{1} p, x^{\prime} p^{\prime}, p_{c}\right), } \\
\left.\varepsilon^{\alpha \beta n p} \frac{\partial S_{\lambda \beta}^{\mathrm{F}}\left(k_{1}, k_{2}\right) p^{\lambda}}{\partial k_{2}^{\alpha}}\right|_{k_{i}=x_{i} p} ^{\mathrm{SGP}} & =\left[\frac{1}{x_{1}-x_{2}+i \varepsilon}\right]^{\text {pole }} \varepsilon^{\alpha \beta n p} \\
\times & {\left[S_{\perp}^{\gamma} \frac{d}{d p_{c}^{\alpha}}+\frac{1}{p \cdot p_{c}}\left\{\left(p \cdot S_{\perp}\right) g_{\alpha}^{\gamma}-S_{\perp \alpha} p^{\gamma}\right\}\right] \widehat{S}_{\beta \gamma}^{\mathrm{F}}\left(x_{1} p, x^{\prime} p^{\prime}, p_{c}\right),(2.5) }
\end{aligned}
$$

where $\varepsilon^{\alpha \beta n p} S_{\perp}^{\gamma} \widehat{S}_{\beta \gamma}^{\mathrm{I}}\left(x_{1} p, x^{\prime} p^{\prime}, p_{c}\right)$ represents the partonic cross section for the $2 \rightarrow 2$ scattering process, $q\left(x_{1} p\right)+b\left(x^{\prime} p^{\prime}\right) \rightarrow q\left(p_{c}\right)+b\left(x_{1} p+x^{\prime} p^{\prime}-p_{c}\right)(b=q$ or $g)$, as shown in Fig. 2 . It is obtained by the spinor projection $\varepsilon^{\alpha \beta n p} \gamma_{5} \gamma_{\beta} x_{1} p$ for the initial parton with momentum $x_{1} p$, the unpolarized projection for the parton $b$ with momentum $x^{\prime} p^{\prime}$, and the projection $\gamma_{5} \delta_{\perp} p_{c}$ for the final parton fragmenting into $H^{\uparrow}$, but has the same color factor as the ISI diagrams in Fig. $1 .^{2}$ Except for the color factor, the twist-2 partonic cross section for the spin-transfer reaction $p^{\uparrow}\left(p, S_{N \perp}\right)+p\left(p^{\prime}\right) \rightarrow$ $H^{\uparrow}\left(P_{h}, S_{\perp}\right)+X$ [18] can be written as $S_{N \perp}^{\beta} S_{\perp}^{\gamma} \widetilde{S}_{\beta \gamma}^{\mathrm{I}}\left(x_{1} p, x^{\prime} p^{\prime}, p_{c}\right) . \widetilde{S}_{\beta \gamma}^{\mathrm{F}}\left(x_{1} p, x^{\prime} p^{\prime}, p_{c}\right)$ in (3.8) is defined similarly but with the color factors for the FSI diagrams in Fig. 1, and thus $\widehat{S}_{\beta \gamma}^{\mathrm{I}}$ and $\widetilde{S}_{\beta \gamma}^{\mathrm{F}}$ differ only in the color factors. We note the appearance of the extra terms in (2.5) for FSI compared with (2.4) for ISI, which is different from the case of the $G_{F}(x, x)$ contribution to $p^{\uparrow} p \rightarrow \pi X$.
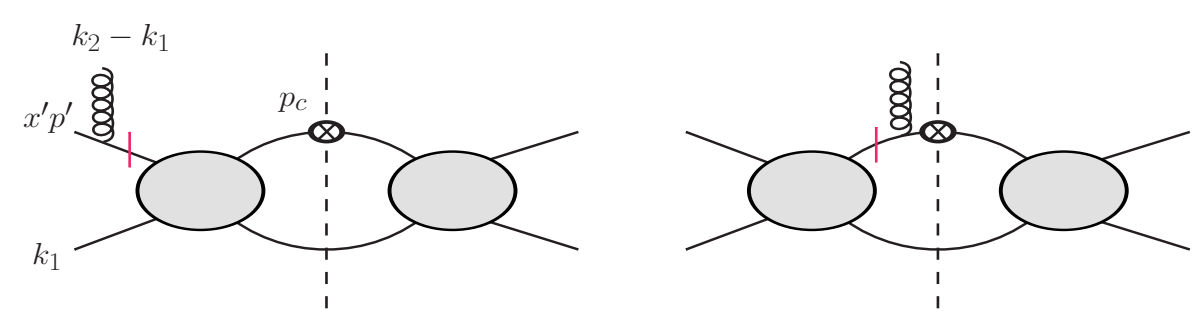

Figure 1: Diagrammatic representation of the hard part for the SGP contribution. Left (Right) diagram corresponds to ISI (FSI) and gives $S_{\lambda \beta}^{\mathrm{I}}\left(k_{1}, k_{2}\right)\left(S_{\lambda \beta}^{\mathrm{F}}\left(k_{1}, k_{2}\right)\right)$. The SGP is given as a pole part of the bared propagator. The circled cross indicates the fragmentation insertion for $H^{\uparrow}$. Each blob represents the $2 \rightarrow 2$ scattering amplitude.

\footnotetext{
${ }^{2}$ We have factored out the spin vector $S_{\perp}^{\gamma}$ to define $\widetilde{S}_{\beta \gamma}^{\mathrm{I}}\left(x_{1} p, x^{\prime} p^{\prime}, p_{c}\right)$.
} 


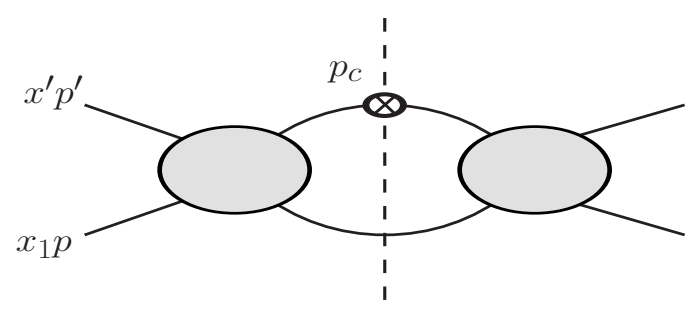

Figure 2: Diagrammatic representation for $\widetilde{S}_{\beta \gamma}^{\mathrm{L}, \mathrm{F}}\left(x_{1} p, x^{\prime} p^{\prime}, p_{c}\right)$.

From (2.4) and (2.5), one can show that the hard factor for the nonderivative terms vanishes and can easily obtain the total SGP contribution as $[10,11]$

$$
P_{h}^{0} \frac{d \Delta \sigma^{\mathrm{SGP}}}{d^{3} P_{h}}=\frac{\pi M_{N} \alpha_{s}^{2}}{s} \varepsilon^{p_{h} p n S_{\perp}} \sum_{a, b, c} \int \frac{d x^{\prime}}{x^{\prime}} f_{1}^{b}\left(x^{\prime}\right) \int \frac{d z}{z^{3}} H_{1}^{c}(z) \int d x \frac{d E_{F}^{a}(x, x)}{d x} \sigma_{a b \rightarrow c} \delta(\hat{s}+\hat{t}+\hat{u}),
$$

where we have supplied the indices $a, b, c$ for each functions: $a, c$ refer to quark and anti-quark flavors and $b$ refers to quark and anti-quark flavors and gluon. The partonic cross section in each channel $\sigma_{a b \rightarrow c}$ is a function of the Mandelstam variables $\hat{s}=\left(x p+x^{\prime} p^{\prime}\right)^{2}, \hat{t}=\left(x p-p_{c}\right)^{2}$ and $\hat{u}=\left(x^{\prime} p^{\prime}-p_{c}\right)^{2}$, and they are given by

$$
\begin{aligned}
\sigma_{q q^{\prime} \rightarrow q} & =\frac{1}{N^{2}} \frac{2 \hat{s}}{\hat{t}^{2}}-\frac{1}{N^{2}} \frac{\hat{s}^{2}}{\hat{t}^{3}}, \quad \sigma_{q q \rightarrow q}=\sigma_{q q^{\prime} \rightarrow q}-\left(\frac{1}{N}+\frac{1}{N^{3}}\right) \frac{\hat{s}}{\hat{t} \hat{u}}+\frac{1}{N^{3}} \frac{\hat{s}^{2}}{\hat{t}^{2} \hat{u}}, \\
\sigma_{q \bar{q}^{\prime} \rightarrow q} & =\left(\frac{N^{2}-2}{N^{2}}\right) \frac{\hat{s}}{\hat{t}^{2}}-\frac{1}{N^{2}} \frac{\hat{s}^{2}}{\hat{t}^{3}}, \quad \sigma_{q \bar{q} \rightarrow q}=\sigma_{q \bar{q}^{\prime} \rightarrow q \bar{q}^{\prime}}+\frac{1}{N^{3}} \frac{1}{t}+\frac{1}{N^{3}} \frac{\hat{s}}{\hat{t}^{2}}, \\
\sigma_{q \bar{q} \rightarrow \bar{q}} & =-\frac{1}{N^{3}} \frac{1}{\hat{u}}+\left(\frac{1}{N}+\frac{1}{N^{3}}\right) \frac{\hat{s}}{\hat{t} \hat{u}}, \\
\sigma_{q g \rightarrow q} & =-\frac{N^{2}}{N^{2}-1} \frac{\hat{u}}{t^{2}}+\frac{1}{N^{2}-1} \frac{1}{\hat{u}}-\frac{1}{N^{2}\left(N^{2}-1\right)} \frac{\hat{s}}{\hat{t} \hat{u}}-\frac{1}{\left(N^{2}-1\right)} \frac{2 \hat{s}^{2}}{\hat{t}^{3}} .
\end{aligned}
$$

We note the master formula (2.4) and (2.5) can be easily extended to the higher order correction to the SGP contribution and will become a useful tool in actual calculation.

We now discuss the SFP contribution. It receives only the nonderivative contribution and is given by [15]

$$
\begin{aligned}
P_{h}^{0} \frac{d \Delta \sigma^{\mathrm{SFP}}}{d^{3} P_{h}}= & \frac{i M_{N}}{64 \pi^{2} s} \int \frac{d x^{\prime}}{x^{\prime}} f_{1}\left(x^{\prime}\right) \int \frac{d z}{z^{2}} H_{1}(z) \\
& \times \int d x_{1} \int d x_{2} E_{F}\left(x_{1}, x_{2}\right) \varepsilon^{\alpha \beta n p}\left(\frac{1}{x_{1}-x_{2}}\right) S_{\alpha \beta}^{\mathrm{SFP}}\left(x_{1} p, x_{2} p\right),
\end{aligned}
$$

where $S_{\alpha \beta}^{\mathrm{SFP}}\left(x_{1} p, x_{2} p\right)$ represents the corresponding hard part. By the direct calculation of the diagrams, it turned out that the SFP contribution completely vanishes in all channels after summing over all the diagrams. ${ }^{3}$ As a result, the derivative term of SGP contribution (2.6) becomes the final cross section formula.

Finally, we note that the contribution from the twist-3 fragmentation for $H^{\uparrow}$ to (1.1) needs to be analyzed, which will be reported elsewhere.

\footnotetext{
${ }^{3}$ The SFP contribution from $E_{F}$ to $p^{\uparrow} p \rightarrow \gamma X$ was also shown to vanish in [17].
} 


\section{3. $A_{L T}$ in hadron production in $p p$ collision}

\subsection{Contribution from twist-3 distribution in the longitudinally polarized proton}

In this section we present the cross section for the second term of (1.3) following [5]. To this end we first define twist-3 distribution functions in the longitudinally polarized nucleon relevant to this contribution. The lightcone quark correlation function defines the transversity distribution $h_{1}(x)$ (in the nucleon $A$ ) and the twist-3 longitudinally polarized distribution $h_{L}(x)$ (in the nucleon $B)$ as [19]

$$
M_{i j}(x)=\int \frac{d \lambda}{2 \pi} e^{i \lambda x}\left\langle P S\left|\bar{\psi}_{j}(0) \psi_{i}(\lambda n)\right| P S\right\rangle=\frac{1}{2}\left(\gamma_{5} \$_{\perp} p\right)_{i j} h_{1}(x)+\frac{M_{N}}{2} \Lambda\left(i \gamma_{5} \sigma^{n p}\right)_{i j} h_{L}(x)+\cdots,(3
$$

where $S$ is the nucleon spin vector normalized as $S^{2}=-1$ and $\Lambda=M_{N}(S \cdot n)$ is its helicity. Twist3 quark-gluon correlation function in the longitudinally polarized nucleon $H_{F L}\left(x_{1}, x_{2}\right)$ is defined as [20]

$$
\begin{aligned}
M_{F i j}^{\alpha}\left(x_{1}, x_{2}\right) & =\int \frac{d \lambda}{2 \pi} \int \frac{d \mu}{2 \pi} e^{i \lambda x_{1}} e^{i \mu\left(x_{2}-x_{1}\right)}\left\langle P S\left|\bar{\psi}_{j}(0) g F^{\alpha n}(\mu n) \psi_{i}(\lambda n)\right| P S\right\rangle \\
& =i \frac{M_{N}}{2} g_{\perp}^{\alpha \beta} \Lambda\left(\gamma_{5} \gamma_{\beta} p\right)_{i j} H_{F L}\left(x_{1}, x_{2}\right)+\cdots
\end{aligned}
$$

where $g_{\perp}^{\alpha \beta}=g^{\alpha \beta}-p^{\alpha} n^{\beta}-p^{\beta} n^{\alpha}$. It follows from hermiticity and $P T$-invariance that $H_{F L}\left(x_{1}, x_{2}\right)=$ $-H_{F L}\left(x_{2}, x_{1}\right)$. We also need another twist-3 distribution $\tilde{h}_{L}(x)$ defined by

$$
\begin{gathered}
M_{\partial i j}^{\alpha}(x)=\lim _{z_{\perp} \rightarrow 0} \int \frac{d \lambda}{2 \pi} e^{i \lambda x} \frac{\partial}{\partial z_{\perp \alpha}}\left\langle P S\left|\bar{\psi}_{j}(0)[0, \infty n]\left[\infty n, \infty n+z_{\perp}\right]\left[\infty n+z_{\perp}, \lambda n+z_{\perp}\right] \psi_{i}\left(\lambda n+z_{\perp}\right)\right| P S\right\rangle \\
=i \frac{M_{N}}{2} g_{\perp}^{\alpha \beta} \Lambda\left(\gamma_{5} \gamma_{\beta} p\right)_{i j} \tilde{h}_{L}(x)+\cdots
\end{gathered}
$$

Although three types of twist-3 distribution functions (3.1), (3.2) and (3.3) appear in the derivation of the cross section, they are not independent, but obey constraint relations by QCD equation of motion (e.o.m.) and the Lorentz invariance property. From QCD e.o.m. one has

$$
h_{L}(x)=-\frac{2}{x} \int_{-1}^{1} d x_{1} \mathscr{P}\left(\frac{1}{x_{1}-x}\right) H_{F L}\left(x_{1}, x\right)-\frac{2}{x} \tilde{h}_{L}(x) .
$$

The operator product expansion gives another relation among $h_{L}(x), h_{1}(x)$, and $H_{F L}\left(x_{1}, x_{2}\right)$ as [20]

$$
-x^{2} \frac{d}{d x}\left(\frac{1}{x} h_{L}(x)\right)=2 h_{1}(x)+2 \int_{-1}^{1} d x_{1} \mathscr{P} \frac{1}{x-x_{1}}\left(\frac{\partial}{\partial x}-\frac{\partial}{\partial x_{1}}\right) H_{F L}\left(x, x_{1}\right) .
$$

The combination of (3.4) and (3.5) leads to

$$
\frac{d \tilde{h}_{L}(x)}{d x}-h_{1}(x)+h_{L}(x)=2 \int_{-1}^{1} d x_{1} \mathscr{P} \frac{1}{\left(x-x_{1}\right)^{2}} H_{F L}\left(x, x_{1}\right),
$$

which is known as a Lorentz invariance relation in the literature [21].

With these twist-3 correlation functions, one can calculate the cross section. Unlike "naively $T$-odd" single spin asymmetry, $A_{L T}$ is " $T$-even", and thus the cross section is caused as a nonpole contribution from the hard part. Following the Feynman gauge formalism for the nonpole 

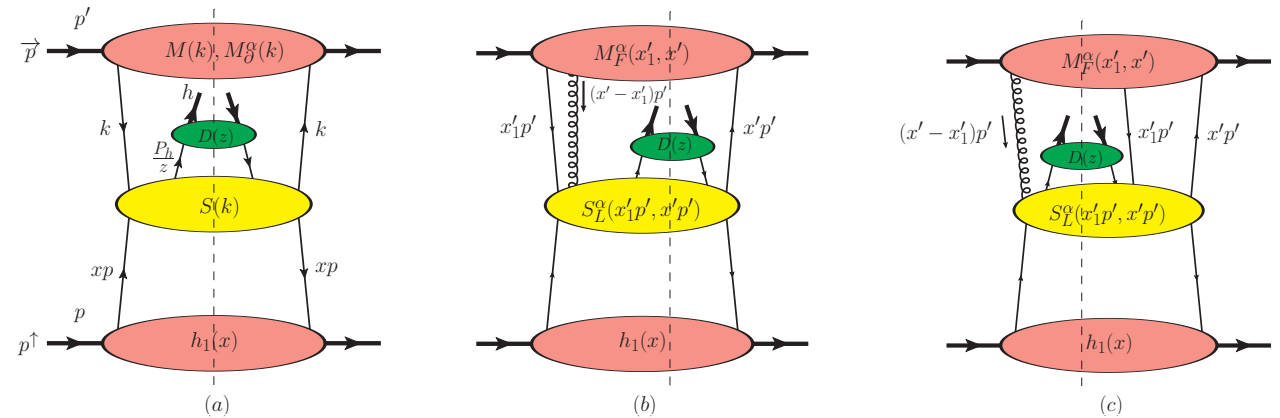

Figure 3: Generic diagrams for the twist-3 distribution contribution from the longitudinally polarized nucleon (upper blob) and the transversity distribution (lower blob) to the process (1.3). Diagram (a) gives rise to the first and second terms in (3.7), and (b) and (c) are for the third term in (3.7). Mirror diagrams of (b) and (c) also contribute, which are included in (3.7).

contribution [22], the twist-3 cross section for the second term of (1.3) is obtained from

$$
\begin{aligned}
& P_{h}^{0} \frac{d \sigma_{L T}^{\text {Long }}}{d^{3} \vec{P}_{h}} \\
& =\frac{1}{16 \pi^{2} s} \int \frac{d x}{x} h_{1}(x) \int \frac{d z}{z^{2}} D(z)\left\{\int d x^{\prime} \operatorname{Tr}\left[M\left(x^{\prime}\right) S\left(x^{\prime} p^{\prime}\right)\right]+i \omega_{\beta}^{\alpha} \int d x^{\prime} \operatorname{Tr}\left[\left.M_{\partial}^{\beta}\left(x^{\prime}\right) \frac{\partial S(k)}{\partial k^{\alpha}}\right|_{k=x^{\prime} p^{\prime}}\right]\right. \\
& \left.\quad+2 i \omega_{\beta}^{\alpha} \int d x^{\prime} \int d x_{1}^{\prime} \mathscr{P} \frac{1}{x_{1}^{\prime}-x^{\prime}} \operatorname{Tr}\left[M_{F}^{\beta}\left(x_{1}^{\prime}, x^{\prime}\right) S_{L \alpha}\left(x_{1}^{\prime} p^{\prime}, x^{\prime} p^{\prime}\right)\right]\right\},
\end{aligned}
$$

where $M\left(x^{\prime}\right), M_{\partial}^{\beta}\left(x^{\prime}\right)$ and $M_{F}\left(x_{1}^{\prime}, x^{\prime}\right)$ are, respectively, defined in (3.1), (3.3) and (3.2) with $p$ and $n$ replaced by $p^{\prime}$ and $n^{\prime}$ similarly defined for the momentum $P^{\prime}$ by the relation $P^{\prime}=p^{\prime}+\left(M_{N}^{2} / 2\right) n^{\prime}$ and $p^{\prime} \cdot n^{\prime}=1$, and $\omega_{\beta}^{\alpha}=g_{\beta}^{\alpha}-p^{\prime \alpha} n_{\beta}^{\prime}$. The partonic hard parts $S(k)$ and $S_{L \alpha}\left(x_{1}^{\prime} p^{\prime}, x^{\prime} p^{\prime}\right)$ are shown by the middle blobs of Fig. 3(a) and Fig. 3(b),(c), respectively. Here $S_{L \alpha}\left(x_{1}^{\prime} p^{\prime}, x^{\prime} p^{\prime}\right)$ represents the hard part for the diagram in which the coherent gluon line from $M_{F}^{\beta}\left(x_{1}^{\prime}, x^{\prime}\right)$ is located in the left of the cut, and the effect of the mirror diagrams are taken into account by the principal value prescription and the factor 2 for the third term in (3.7). Calculating the lowest order Feynman diagrams for the hard part in (3.7), one can obtain the cross section in terms of $h_{L}, \tilde{h}_{L}$ and $H_{F L}$. We further found that the simple structure of the partonic hard cross section for $H_{F L}$ allows us to eliminate it by using (3.6). This way we eventually obtain the cross section for the second term of (1.3) as [5] $]^{4}$

$$
\begin{aligned}
& P_{h}^{0} \frac{d \sigma_{L T}^{\text {Long }}}{d^{3} \vec{P}_{h}}=\frac{2 \alpha_{s}^{2} M_{N} \Lambda}{S}\left(S_{\perp} \cdot p_{h}\right) \sum_{i} \sum_{a, b, c} \int_{0}^{1} \frac{d x}{x} h_{1}^{a}(x) \int_{0}^{1} \frac{d z}{z^{3}} D^{c}(z) \int_{0}^{1} d x^{\prime} \delta(\hat{s}+\hat{t}+\hat{u}) \\
& \times\left[h_{1}^{b}\left(x^{\prime}\right) \hat{\sigma}_{1}^{i}+h_{L}^{b}\left(x^{\prime}\right) \hat{\sigma}_{2}^{i}+\frac{d \tilde{h}_{L}^{b}\left(x^{\prime}\right)}{d x^{\prime}} \hat{\sigma}_{3}^{i}\right]
\end{aligned}
$$

where $\hat{\sigma}_{1,2,3}^{i}$ represent the partonic hard cross section in the channel $i$, and $a, b, c$ refer to parton species $(a, b=q, \bar{q}$, and $c=q, \bar{q}, g)$. The Mandelstam variables in the partonic level are defined as

\footnotetext{
${ }^{4}$ In this section, $p_{h}$ refers to the final hadron momentum defined similarly to the hyperon case in the previous section.
} 
$\hat{s}=\left(x p+x^{\prime} p^{\prime}\right)^{2}, \hat{t}=\left(x p-p_{c}\right)^{2}$ and $\hat{u}=\left(x^{\prime} p^{\prime}-p_{c}\right)^{2}$. The partonic cross section in each channel is given as follows:

(i) $q q \rightarrow q q$ channel:

$$
\hat{\sigma}_{1}=-\frac{1}{N^{3}} \frac{\hat{t}-\hat{u}}{\hat{t} \hat{u}}, \quad \hat{\sigma}_{2}=\left(\frac{1}{N}+\frac{1}{N^{3}}\right) \frac{\hat{t}-\hat{u}}{2 \hat{t} \hat{u}}, \quad \hat{\sigma}_{3}=-\frac{1}{N} \frac{1}{\hat{t}}+\frac{1}{N^{3}} \frac{1}{\hat{u}} .
$$

(ii) $\bar{q} q \rightarrow q^{\prime} \bar{q}^{\prime}$ channel:

$$
\hat{\sigma}_{1}=\frac{\hat{t}}{\hat{s}^{2}}+\frac{1}{N^{2}} \frac{2}{\hat{s}}, \quad \hat{\sigma}_{2}=-\frac{\hat{u}}{\hat{s}^{2}}+\frac{1}{N^{2}} \frac{2 \hat{u}-\hat{s}}{\hat{s}^{2}}, \quad \hat{\sigma}_{3}=\frac{1}{\hat{s}}+\frac{1}{N^{2}} \frac{\hat{u}-2 \hat{s}}{\hat{s}^{2}} .
$$

(iii) $\bar{q} q \rightarrow \bar{q}^{\prime} q^{\prime}$ channel:

$$
\hat{\sigma}_{1}=-\frac{\hat{u}}{\hat{s}^{2}}-\frac{1}{N^{2}} \frac{2}{\hat{s}}, \quad \hat{\sigma}_{2}=\frac{\hat{t}}{\hat{s}^{2}}+\frac{1}{N^{2}} \frac{\hat{s}-2 \hat{t}}{\hat{s}^{2}}, \quad \hat{\sigma}_{3}=\frac{1}{N^{2}} \frac{2 \hat{s}+\hat{u}}{\hat{s}^{2}} .
$$

(iv) $\bar{q} q \rightarrow q \bar{q}$ channel:

$$
\begin{aligned}
& \hat{\sigma}_{1}=\frac{\hat{t}}{\hat{s}^{2}}+\frac{1}{N^{2}} \frac{2}{\hat{s}}+\frac{1}{N} \frac{1}{\hat{s}}-\frac{1}{N^{3}} \frac{1}{\hat{u}}, \quad \hat{\sigma}_{2}=-\frac{\hat{u}}{\hat{s}^{2}}+\frac{1}{N^{2}} \frac{2 \hat{u}-\hat{s}}{\hat{s}^{2}}-\frac{1}{N} \frac{\hat{t}}{2 \hat{s} \hat{u}}-\frac{1}{N^{3}} \frac{\hat{t}+4 \hat{u}}{2 \hat{s} \hat{u}}, \\
& \hat{\sigma}_{3}=\frac{1}{\hat{s}}+\frac{1}{N^{2}} \frac{\hat{u}-2 \hat{s}}{\hat{s}^{2}}-\frac{1}{N^{3}} \frac{\hat{u}-\hat{s}}{\hat{s} \hat{u}} .
\end{aligned}
$$

(v) $\bar{q} q \rightarrow \bar{q} q$ channel:

$$
\begin{aligned}
& \hat{\sigma}_{1}=-\frac{\hat{u}}{\hat{s}^{2}}-\frac{1}{N^{2}} \frac{2}{\hat{s}}-\frac{1}{N} \frac{1}{\hat{s}}+\frac{1}{N^{3}} \frac{1}{\hat{t}}, \quad \hat{\sigma}_{2}=\frac{\hat{t}}{\hat{s}^{2}}+\frac{1}{N^{2}} \frac{\hat{s}-2 \hat{t}}{\hat{s}^{2}}+\frac{1}{N} \frac{\hat{u}}{2 \hat{s} \hat{t}}+\frac{1}{N^{3}} \frac{4 \hat{t}+\hat{u}}{2 \hat{s} \hat{t}}, \\
& \hat{\sigma}_{3}=\frac{1}{N^{2}} \frac{2 \hat{s}+\hat{u}}{\hat{s}^{2}}-\frac{1}{N} \frac{1}{\hat{t}}+\frac{1}{N^{3}} \frac{1}{\hat{s}} .
\end{aligned}
$$

(vi) $\bar{q} q \rightarrow g g$ channel:

$$
\begin{aligned}
& \hat{\sigma}_{1}=C_{F} \frac{2\left(\hat{t}^{3}-\hat{u}^{3}\right)}{\hat{s}^{2} \hat{t} \hat{u}}-\frac{1}{N} \frac{\hat{t}-\hat{u}}{\hat{s}^{2}}, \quad \hat{\sigma}_{2}=-C_{F} \frac{2(\hat{t}-\hat{u})\left(\hat{s}^{2}+\hat{t} \hat{u}\right)}{\hat{s}^{2} \hat{t} \hat{u}}+\frac{C_{F}^{2}}{N} \frac{2(\hat{t}-\hat{u})}{\hat{t} \hat{u}}+\frac{1}{N} \frac{\hat{t}-\hat{u}}{\hat{s}^{2}}, \\
& \hat{\sigma}_{3}=C_{F} \frac{2\left(\hat{t}^{2}-\hat{t} \hat{u}-\hat{u}^{2}\right)}{\hat{s} \hat{t} \hat{u}}-\frac{C_{F}^{2}}{N} \frac{4}{\hat{t}}+\frac{1}{N} \frac{\hat{t}-\hat{u}}{\hat{s}^{2}} .
\end{aligned}
$$

For the charge conjugated channels (where an antiquark comes from the longitudinally polarized proton) we find $\hat{\sigma}_{\bar{a} \bar{b} \rightarrow \bar{c} \bar{d}}=\hat{\sigma}_{a b \rightarrow c d}$, where $\hat{\sigma}_{a b \rightarrow c d}$ are given in Eqs. (3.9)-(3.14). Since there are two independent relations, (3.4) and (3.6), $h_{L}\left(x^{\prime}\right)$ and $\tilde{h}_{L}\left(x^{\prime}\right)$ can be expressed in terms of $H_{F L}\left(x_{1}^{\prime}, x^{\prime}\right)$ and the transversity distribution $h_{1}\left(x^{\prime}\right)$, and thus are "auxiliary" twist-3 distributions. ${ }^{5}$ However, the simple structure of the partonic cross section for $H_{F L}\left(x_{1}^{\prime}, x^{\prime}\right)$ led to Eq. (3.8) for the LO cross section, which contains only one-variable functions.

\subsection{Contribution from twist-3 fragmentation}

In this section we present the LO cross section for the third term of (1.3) following our recent paper [6]. We first define twist-3 fragmentation functions necessary in the derivation. The quarkquark matrix element gives two real twist-3 fragmentation functions for $q \rightarrow h$, which read

$$
\begin{aligned}
\Delta^{q}(z)= & \frac{1}{N} \sum_{X} \int \frac{d \lambda}{2 \pi} e^{-i \frac{\lambda}{z}}\left\langle 0\left|\psi_{i}^{q}(0)\right| P_{h} ; X\right\rangle\left\langle P_{h} ; X\left|\bar{\psi}_{j}^{q}(\lambda w)\right| 0\right\rangle \\
= & \frac{M_{N}}{z} \delta_{i j} \hat{e}_{1}^{h / q}(z)+\frac{M_{N}}{2 z}\left(\sigma_{\lambda \alpha} i \gamma_{5}\right)_{i j} \varepsilon^{\lambda \alpha w p_{h}} \hat{e}_{\overline{1}}^{h / q}(z)+\cdots,
\end{aligned}
$$

\footnotetext{
${ }^{5}$ We refer the reader to Ref. [21] for an extensive work on relations between twist- 3 functions (including fragmentation ones) and their importance in showing the Lorentz invariance of twist- 3 cross sections.
} 
where we introduced the nucleon mass $M_{N}$ to make the functions dimensionless. The lightlike vectors $p_{h}^{\mu}$ and $w^{\mu}$ are defined after Eq. (2.2). Next, we introduce the so-called $F$-type quarkgluon-quark twist-3 fragmentation functions. We can define two independent functions as

$$
\begin{aligned}
\Delta_{F}^{q, \alpha}\left(z_{1}, z\right) & =\frac{1}{N} \sum_{X} \int \frac{d \lambda}{2 \pi} \int \frac{d \mu}{2 \pi} e^{-i \frac{\lambda}{z_{1}}} e^{-i \mu\left(\frac{1}{z}-\frac{1}{z_{1}}\right)}\left\langle 0\left|\psi_{i}^{q}(0)\right| P_{h} ; X\right\rangle\left\langle P_{h} ; X\left|\bar{\psi}_{j}^{q}(\lambda w) g F^{\alpha w}(\mu w)\right| 0\right\rangle \\
& =\frac{M_{N}}{2 z}\left(\gamma_{5} p_{h} \gamma_{\lambda}\right)_{i j} \varepsilon^{\lambda \alpha w p_{h}} \widehat{E}_{F}^{h / q}\left(z_{1}, z\right)+\cdots \\
\widetilde{\Delta}_{F}^{q, \alpha}\left(z_{1}, z\right) & =\frac{1}{N} \sum_{X} \int \frac{d \lambda}{2 \pi} \int \frac{d \mu}{2 \pi} e^{-i \frac{\lambda}{z_{1}}} e^{-i \mu\left(\frac{1}{z}-\frac{1}{z_{1}}\right)}\left\langle 0\left|\bar{\psi}_{j}^{q}(\lambda w) \psi_{i}^{q}(0)\right| P_{h} ; X\right\rangle\left\langle P_{h} ; X\left|g F^{\alpha w}(\mu w)\right| 0\right\rangle \\
& =\frac{M_{N}}{2 z}\left(\gamma_{5} p_{h} \gamma_{\lambda}\right)_{i j} \varepsilon^{\lambda \alpha w p_{h}} \widetilde{E}_{F}^{h / q}\left(z_{1}, z\right)+\cdots
\end{aligned}
$$

We note that both $\widehat{E}_{F}^{h / q}\left(z_{1}, z\right)$ and $\widetilde{E}_{F}^{h / q}\left(z_{1}, z\right)$ in general are complex functions. The correlator $\widehat{E}_{F}^{h / q}\left(z_{1}, z\right)$ has support on $1>z>0$ and $z_{1}>z$, while $\widetilde{E}_{F}^{h / q}\left(z_{1}, z\right)$ has support on $\frac{1}{z}-\frac{1}{z_{1}}>1, \frac{1}{z_{1}}<0$, and $\frac{1}{z}>0[22,23]$. To derive the cross section, we also need the kinematical twist- 3 fragmentation function $\widetilde{e}^{h / q}(z)$ as

$$
\begin{aligned}
\Delta_{\partial}^{q, \alpha}(z)= & \frac{1}{N} \sum_{X} \int \frac{d \lambda}{2 \pi} e^{-i \frac{\lambda}{z}}\left\langle 0\left|[\infty w, 0] \psi_{i}^{q}(0)\right| P_{h} ; X\right\rangle\left\langle P_{h} ; X\left|\bar{\psi}_{j}^{q}(\lambda w)[\lambda w, \infty w]\right| 0\right\rangle \overleftarrow{\partial}^{\alpha} \\
& =\frac{M_{N}}{2 z}\left(\gamma_{5} p_{h} \gamma_{\lambda}\right)_{i j} \varepsilon^{\lambda \alpha w p_{h}} \widehat{e}^{h / q}(z)+\cdots .
\end{aligned}
$$

Owing to the QCD e.o.m., there is a constraint relation among the twist-3 fragmentation functions:

$$
z \int_{z}^{\infty} \frac{d z_{1}}{z_{1}^{2}} P\left(\frac{1}{1 / z_{1}-1 / z}\right) \widehat{E}_{F}^{h / q}\left(z_{1}, z\right)+z \widehat{e}^{h / q}(z)=\widehat{e}_{1}^{h / q}(z)+i \widehat{e}_{\overline{1}}^{h / q}(z) .
$$

The real and imaginary parts of this relation respectively give

$$
\begin{array}{r}
z \int_{z}^{\infty} \frac{d z_{1}}{z_{1}^{2}} P\left(\frac{1}{1 / z_{1}-1 / z}\right) \widehat{E}_{F}^{h / q, \Re}\left(z_{1}, z\right)=\widehat{e}_{1}^{h / q}(z), \\
z \int_{z}^{\infty} \frac{d z_{1}}{z_{1}^{2}} P\left(\frac{1}{1 / z_{1}-1 / z}\right) \widehat{E}_{F}^{h / q, \mathfrak{I}}\left(z_{1}, z\right)+z \widetilde{e}^{h / q, \mathfrak{I}}(z)=\widehat{e}_{\overline{1}}^{h / q}(z),
\end{array}
$$

where $\mathfrak{R}(\mathfrak{I})$ indicates the real (imaginary) part of the function. It was shown that Eq. (3.21) ensures the gauge invariance of the polarized cross section formula in the case of the transverse single spin asymmetry in semi-inclusive deep inelastic scattering [22]. Likewise Eq. (3.20) plays the same role in the case of $A_{L T}$ in proton-proton collisions.

Applying the Feynman gauge formalism of [22], the twist-3 cross section for the third line of (1.3) can be obtained from the following formula:

$$
\begin{aligned}
P_{h}^{0} \frac{d \sigma_{L T}^{\mathrm{Frag}}}{d^{3} \vec{P}_{h}} & =\frac{1}{16 \pi^{2} S} \Lambda \sum_{a, b} \int \frac{d x}{x} h_{1}^{a}(x) \int \frac{d x^{\prime}}{x^{\prime}} g_{1}^{b}\left(x^{\prime}\right) \\
& \times\left\{\int \frac{d z}{z^{2}} \operatorname{Tr}\left[\Delta^{a}(z) S\left(\frac{p_{h}}{z}\right)\right]+\int \frac{d z}{z^{2}} \operatorname{Im} \operatorname{Tr}\left[\left.\Omega_{\beta}^{\alpha} \Delta_{\partial}^{a, \beta}(z) \frac{\partial S(k)}{\partial k^{\alpha}}\right|_{k=p_{h} / z}\right]\right.
\end{aligned}
$$




$$
\begin{aligned}
& +2 \int \frac{d z_{1}}{z_{1}^{2}} \int \frac{d z_{2}}{z_{2}^{2}}\left(\frac{1}{1 / z_{1}-1 / z_{2}}\right) \operatorname{Im} \operatorname{Tr}\left[\Omega_{\beta}^{\alpha} \Delta_{F}^{a, \beta}\left(z_{1}, z_{2}\right) S_{L \alpha}\left(\frac{p_{h}}{z_{1}}, \frac{p_{h}}{z_{2}}\right)\right] \\
& \left.+2 \int \frac{d z_{1}}{z_{1}^{2}} \int \frac{d z_{2}}{z_{2}^{2}}\left(\frac{1}{1 / z_{1}-1 / z_{2}}\right) \operatorname{Im} \operatorname{Tr}\left[\Omega_{\beta}^{\alpha} \widetilde{\Delta}_{F}^{a, \beta}\left(z_{1}, z_{2}\right) \widetilde{S}_{L \alpha}\left(\frac{p_{h}}{z_{1}}, \frac{p_{h}}{z_{2}}\right)\right]\right\},
\end{aligned}
$$

where $g_{1}^{b}\left(x^{\prime}\right)$ is the twist-2 helicity distribution, $\Omega_{\beta}^{\alpha}=g_{\beta}^{\alpha}-p_{h}^{\alpha} w_{\beta}, S(k)$ is the partonic hard part for $\Delta^{q}(z)$, and $S_{L \alpha}\left(k_{1}, k_{2}\right)$ and $\widetilde{S}_{L \alpha}\left(k_{1}, k_{2}\right)$ are, respectively, the hard parts for $\Delta_{F}^{q, \alpha}\left(z_{1}, z_{2}\right)$ and $\widetilde{\Delta}_{F}^{q, \alpha}\left(z_{1}, z_{2}\right)$. Calculation of the lowest-order Feynman diagrams for the hard part eventually gives the cross section as [6]

$$
P_{h}^{0} \frac{d \sigma_{L T}^{\mathrm{Frag}}}{d^{3} \vec{P}_{h}}=-\frac{2 \alpha_{S}^{2} M_{N}}{S} \Lambda\left(p_{h} \cdot S_{\perp}\right) \sum_{i} \sum_{a, b, c} \int_{0}^{1} \frac{d z}{z^{4}} \hat{e}_{1}^{h / c}(z) \int_{0}^{1} \frac{d x^{\prime}}{x^{\prime}} g_{1}^{b}\left(x^{\prime}\right) \int_{0}^{1} \frac{d x}{x} h_{1}^{a}(x) \hat{\sigma}_{i} \delta(\hat{s}+\hat{t}+\hat{u}),
$$

where $\hat{\sigma}_{i}$ refers to the partonic hard factor in channel $i$. In deriving (3.23), we have again eliminated $\widehat{E}_{F}^{h / q}\left(z_{1}, z\right)$ in favor of $\hat{e}^{h / q}(z)$ by using (3.20). This was possible because the partonic hard cross section for the former has a simple structure. The hard factors $\hat{\sigma}_{i}$ are given by

$$
\begin{array}{ll}
\hat{\sigma}_{q g \rightarrow q g}=-\frac{3}{2}\left[\frac{\hat{s}^{2}+\hat{u}^{2}}{\hat{t}^{2} \hat{u}}-\frac{1}{N^{2}} \frac{1}{\hat{u}}\right], & \hat{\sigma}_{q q^{\prime} \rightarrow q q^{\prime}}=\frac{2 C_{F}}{N} \frac{\hat{s}}{\hat{t}^{2}}, \\
\hat{\sigma}_{q q \rightarrow q q}=\frac{2 C_{F}}{N}\left[\frac{\hat{s}}{\hat{t}^{2}}+\frac{1}{N} \frac{\hat{s}(\hat{s}-2 \hat{t})}{2 \hat{t}^{2} \hat{u}}\right], & \hat{\sigma}_{q \bar{q} \rightarrow q \bar{q}}=\frac{2 C_{F}}{N}\left[\frac{\hat{s}}{\hat{t}^{2}}+\frac{1}{N} \frac{\hat{u}-2 \hat{t}}{2 \hat{t}^{2}}\right], \\
\hat{\sigma}_{q \bar{q}^{\prime} \rightarrow q \bar{q}^{\prime}}=\hat{\sigma}_{q q^{\prime} \rightarrow q q^{\prime}}, & \hat{\sigma}_{\bar{q} q \rightarrow q \bar{q}}=\frac{2 C_{F}}{N^{2}} \frac{3}{2 \hat{u}},
\end{array}
$$

where $C_{F}=\left(N^{2}-1\right) / 2 N$. For the antiquark fragmentation channels we find (c.f. [24]) $\hat{\sigma}_{\bar{a} \bar{b} \rightarrow \bar{c} \bar{d}}=$ $\hat{\sigma}_{a b \rightarrow c d}$, where $\hat{\sigma}_{a b \rightarrow c d}$ are given in (3.24). We also calculated the polarized cross section in lightcone gauge using the procedure of Ref. [24] and found agreement with Eqs. (3.23), (3.24).

\section{Summary}

In this paper, we have studied the hyperon polarization in unpolarized $p p$ collisions and the longitudinal-transverse double spin asymmetry $A_{L T}$ for hadron production in $p p$ collisions. Hyperon polarization is a naively $T$-odd observable, while $A_{L T}$ is a naively $T$-even one. Owing to this fact, the structure of the corresponding twist- 3 cross sections is inherently different, and they can play complementary roles to study hadron structure and test validity of the formalism. We hope that these important spin asymmetries will be measured by the ongoing experiment of the Relativistic Heavy Ion Collider at BNL.

\section{Acknowledgments}

This work has been supported by the Grant-in-Aid for Scientific Research from the Japanese Society of Promotion of Science under Contract No. 26287040 (Y.K.), by the U.S. Department of Energy, Office of Science, Office of Nuclear Physics within the framework of the TMD Topical Collaboration (D.P.), and in part by the NSFC under Grant No. 11575070 (S.Y.). 


\section{References}

[1] G. Bunce et al., Phys. Rev. Lett. 36, 1113 (1976).

[2] G. L. Kane, J. Pumplin and W. Repko, Phys. Rev. Lett. 41, 1689 (1978).

[3] Y. Koike, K. Yabe and S. Yoshida, Phys. Rev. D 92 (2015) no.9, 094011 [arXiv:1509.06830 [hep-ph]].

[4] A. Metz, D. Pitonyak, A. Schäfer and J. Zhou, Phys. Rev. D 86, 114020 (2012) [arXiv:1210.6555 [hep-ph]].

[5] Y. Koike, D. Pitonyak and S. Yoshida, Phys. Lett. B 759 (2016) 75 [arXiv:1603.07908 [hep-ph]].

[6] Y. Koike, D. Pitonyak, Y. Takagi and S. Yoshida, Phys. Lett. B 752 (2016) 95 [arXiv:1508.06499 [hep-ph]].

[7] Y. Koike, K. Tanaka and S. Yoshida, Phys. Lett. B 668, 286 (2008) [arXiv:0805.2289 [hep-ph]].

[8] K. Kanazawa, Y. Koike, A. Metz and D. Pitonyak, Phys. Rev. D 89, 111501(R) (2014) [arXiv:1404.1033 [hep-ph]].

[9] Y. Kanazawa and Y. Koike, Phys. Lett. B 478, 121 (2000) [hep-ph/0001021].

[10] Y. Kanazawa and Y. Koike, Phys. Rev. D 64, 034019 (2001) [hep-ph/0012225].

[11] J. Zhou, F. Yuan and Z.-T. Liang, Phys. Rev. D 78, 114008 (2008). [arXiv:0808.3629[hep-ph]]

[12] C. Kouvaris, J. W. Qiu, W. Vogelsang and F. Yuan, Phys. Rev. D 74, 114013 (2006) [arXiv:hep-ph/0609238].

[13] Y. Koike and K. Tanaka, Phys. Rev. D 76, 011502 (2007) [hep-ph/0703169].

[14] J.-w. Qiu and G. F. Sterman, Phys. Rev. Lett. 67, 2264 (1991); Nucl. Phys. B 378, 52 (1992).

[15] H. Eguchi, Y. Koike and K. Tanaka, Nucl. Phys. B 763, 198 (2007) [arXiv:hep-ph/0610314].

[16] Y. Koike and K. Tanaka, Phys. Lett. B 646, 232 (2007) [Erratum-ibid. B 668, 458 (2008)] [hep-ph/0612117].

[17] K. Kanazawa, Y. Koike, A. Metz and D. Pitonyak, Phys. Rev. D 91, no. 1, 014013 (2015) [arXiv:1410.3448 [hep-ph]].

[18] M. Stratmann and W. Vogelsang, Phys. Lett. B 295, 277 (1992).

[19] R. L. Jaffe and X. D. Ji, Phys. Rev. Lett. 67, 552 (1991); Nucl. Phys. B 375, 527 (1992).

[20] J. Kodaira and K. Tanaka, Prog. Theor. Phys. 101, 191-242 (1999) [hep-ph/9812449 [hep-ph]].

[21] K. Kanazawa, Y. Koike, A. Metz, D. Pitonyak and M. Schlegel, Phys. Rev. D 93, 054024 (2016) [arXiv:1512.07233 [hep-ph]].

[22] K. Kanazawa and Y. Koike, Phys. Rev. D 88, 074022 (2013) [arXiv:1309.1215 [hep-ph]].

[23] S. Meissner and A. Metz, Phys. Rev. Lett. 102, 172003 (2009) [arXiv:0812.3783 [hep-ph]].

[24] A. Metz and D. Pitonyak, Phys. Lett. B 723, 365 (2013) [arXiv:1212.5037 [hep-ph]]. 